\title{
Reopening Sleep Medicine Services in the Conundrum of an Ongoing COVID-19 Pandemic: A Global View
}

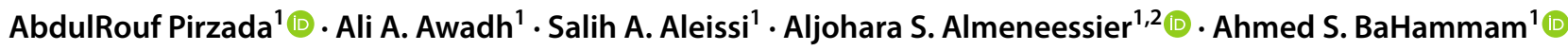

Received: 24 June 2020 / Accepted: 23 July 2020 / Published online: 31 July 2020

(c) Springer Nature Singapore Pte Ltd. 2020

\begin{abstract}
The emergence of COVID-19 brought all healthcare services around the globe to immense strain; hospitals abandoned elective care for acute care. Like all other elective services, sleep medicine services suffered a partial deadlock due to the closing down of the sleep disorders diagnostic and therapeutic services, although clinical consultations and follow-ups, carried on remotely, allowed some mitigation. Since there is dire need to resume the services, we tried to formulate the principles and guidelines to work in this exigent healthcare setting. Principles and guidelines are based on epidemiological and infection control guidelines besides recommendations of various healthcare organizations and sleep societies, after a requisite web search to extract the data.
\end{abstract}

Keywords SARS-Co-V-2 $\cdot$ Sleep disorders $\cdot$ CPAP $\cdot$ Coronavirus $\cdot$ Polysomnography $\cdot$ Home sleep apnea testing

\section{Introduction}

In December 2019, Wuhan China reported sporadic cases of unusual pneumonia. The sporadic phenomenon was transformed rapidly into an epidemic; subsequent global spread prompted the World Health Organization (WHO) to declare it a pandemic [1-3]. Healthcare systems were pushed to the wall; hospitals suspended all elective appointments, procedures, and investigations. Hospital services were stretched to the limits to attend a massive volume of patients; many of them were very sick. Hospitals had to work with staff rationing to prevent the spread in the community and amongst healthcare workers, besides ensuring contingency strength. The out-patient services were taken over by telemedicine clinics, virtual clinics, or telephonic clinics. At most of the centers, resources were shifted from elective care to acute care. Being an elective service in hospitals

Aljohara S. Almeneessier

aljoharas@yahoo.com

$\triangle$ Ahmed S. BaHammam

ashammam2@gmail.com

1 Department of Medicine, College of Medicine, University Sleep Disorders Center, King Saud University, Riyadh, Saudi Arabia

2 Family and Community Medicine Department, College of Medicine, King Saud University, Riyadh, Saudi Arabia and medical centers, sleep medicine services were quick to adapt to the new demands, providing virtual clinic services and telephonic follow-ups, besides disbursing electronic prescriptions. However, sleep studies were suspended, both in-house as well as Home Sleep Apnea Testing (HSAT), resulting in delayed diagnosis and management of patients with various sleep disorders, including high-risk patients like obesity hypoventilation syndrome [4]. Moreover, sleep therapeutic studies are high aerosol-generating procedures, which warrant extra careful handling and thorough infection control guidelines implementation [5, 6]. Nonetheless, optimal treatment of sleep-disordered breathing has been shown to reduce the mortality and morbidity [7]. The outcome of COVID-19 patients is adversely influenced by comorbidities that are also common in patients with sleepdisordered breathing. Thus, it is imperative to manage these sleep-related breathing disorders with urgency rather than postponing them to mitigate sleep problems and to have a favorable influence on COVID-19 outcomes [7]. Moreover, the pandemic is taking a protracted unpredictable course; therefore, we need to devise ways and means to carry on optimal services despite it.

Although the initial response was to curb the rapid spread, enhance preparedness, and ensure resources. Healthcare systems need to chalk out a comprehensive plan to resume almost all the hospital-based services without compromising the well-being of patients and healthcare workers since 
the pandemic is taking a protracted course [4]. Hence the framework of the plan is to minimize the spread of the infection with the simultaneous resumption of services, which warrants a multi-prong strategy taking into account the epidemiology and infection control protocols.

We used different words and phrases like "COVID-19 infection control", "COVID-19 and Sleep services", Sleep medicine", "pandemic", "COVID-19 epidemiology and control", "resumption of sleep medicine services during COVID-19" for the online search to extract data from various societies, organizations, institutions, and experts using multiple databases like PubMed/MEDLINE, Scopus, ISI/ Web of Science, and Google Scholar, in addition to searching the websites of various healthcare organizations and sleep societies.

This review aimed to formulate a practical approach, based on the currently available data, to resume in-laboratory sleep studies and other sleep medicine related services. The right step in this direction is to ensure that the staff of the sleep disorders centers are well-versed in infection control guidelines and protocols, besides, appraisals, and updates about these guidelines and protocols for COVID-19.

Therefore, we need to ensure and support efforts for the gradual and safe resumption of sleep medicine services to the pre-pandemic level without compromising the implementation of best practice guidelines and protocols for infection prevention and control. The exposure of patients and healthcare workers to the virus is to be prevented with the judicious use of personal protective equipment (PPE). We need to maintain continuity of care to avoid loss of follow-up and, at the same time, minimize unnecessary consultations/attendance. Moreover, public health safety, patient concerns, and demands are to be addressed professionally.

\section{General Considerations, Background Preparations, and Useful Principles for the Resumption of Services}

The epidemiological status of the province/city, patient screening (through questionnaires and testing), and preparedness of the center form the structure's backbone and should be considered when making a decision about resuming sleep medicine services [2, 3, 8]. Patient status through screening is defined by the local infection control guidelines or guidelines proposed by validated infection control societies. Patients are identified as: status unknown, presumed negative, self-quarantined, presumed positive, and presumed negative accordingly $[8,9]$. Center preparedness encompasses logistics (healthcare resources) and staff education and training to provide the optimal services without compromising infection control guidelines [8, 10-13]. Table 1 presents a summary of general considerations and background preparation and principles.
Table 1 A summary of general considerations and background preparation and principles for the resumption of clinical services
Background target and principles and goals of preparedness
A. Epidemiological segregation
1. High endemic area or the area with large-scale community transmission
2. Sporadic area or the area with minimal to moderate community transmission
3. Low endemic area or area with no or minimal community transmission
B. Patient status through screening
1. Prescreening
Review (remotely) of any COVID-19 symptoms, travel history, contact history
COVID-19 status (if lab testing was done)
2. Screening at the time of appointment
I. In-person review of any COVID-19 symptoms, travel history, contact history, and
II.COVID-19 status (if lab test was done)
C. Sleep disorders center targets
1. Staff screening
I. Review of symptoms, travel history, contact history
II. COVID-19 status (if lab test was done)
2. Infection control
I. Staff training for maintaining infection control protocol within the center and for the used equipment
II. Staff training for patient identification and risk stratification
3. Logistics
I. Maintaining a requisite supply of full PPE and chemicals for disinfection
II. Maintaining coordination for epidemiological updates 


\subsection{Epidemiological Status}

The epidemiological status of the region or province provides first-hand information about the infection burden in the community; thus, it is the first step in decision making $[3,14]$. The regions/provinces/cities are classified as: a high epidemic area or an area with a large scale community transmission, a sporadic area or an area with minimal to moderate community transmission (sporadic cases or small clusters), and a low endemic area where community transmission is absent or minimal $[2,8]$. The general agreement is to follow the local, regional, and international epidemiological situational reports on an everyday basis to commence sleep studies, both diagnostic and therapeutic $[2,8,15,16]$.

\subsection{COVID-19 Status}

The second step is to determine the COVID-19 status of patients. Status determination tool compromises a questionnaire, a comprehensive interrogative method to know about patient clinical history, contact history, location history, travel history, diagnostic history (COVID-19 testing), and laboratory testing. The same must be applied to staff to deem them fit to carry out the job. Thus, screening must be a practice, pre-procedural, and at the time of the procedure $[2,8,11,16]$.

\section{Screening}

Screening is recommended for patients as well as staff, and sometimes on rare occasions, for patient attendants.

\subsection{Patient Screening Protocols}

It is based on pre-appointment and time of appointment screening.

\subsubsection{Pre-Screening (Prior to Appointment)}

Use phone calls, patient portals, virtual meetings, or online self-assessment tools (to be delivered online and retrieved online). Review COVID-19 symptoms (such as fever, cough, or shortness of breath), review COVID-19 status, and reschedule patients with symptoms or positive or pending COVID-19 tests $[8,10,11,13]$.

\subsubsection{Screening at the time of appointment}

A screening protocol needs to be repeated once a patient attends the clinic in person for the procedure, i.e., inquiring about the symptoms, contact history, travel history, and COVID-19 status besides taking the temperature $[2,8,10$, $17,18]$.

\subsection{Screening of Staff}

The same screening protocol is to be applied to the healthcare personnel, including the office staff. They must be routinely screened for any COVID-19 symptoms, contact history, and travel history (preferably via questionnaire every day). They should be encouraged to volunteer any information about symptoms and should take temperature checks twice per day. Any staff with symptoms should be sent home. Staff rationing should be adopted to ensure minimum exposure and to leave room for sickness or quarantine deficits $[8,16-18]$.

Sick leave policies of the sleep disorders center need to be revisited to make them flexible and non-punitive for new demands and contingency. Allow all ill personnel to stay home and encourage the staff not to report to work when they are ill $[8,16-18]$.

\subsection{Screening with Point-of-Contact Testing}

Whenever adequate COVID-19 diagnostic testing is available, patients should be tested in a reasonable timeframe before the appointment (i.e., doing the test when the result will have a bearing upon the appointment, not doing it in a long time frame so that the result becomes irrelevant). The sleep disorders center healthcare personnel, cleaning staff, and other hospital staff (visiting the sleep disorders center) should be regularly screened with a diagnostic test when COVID-19 testing is available and logistically possible [3, $8,16]$. Whenever there is reasonable doubt about exposure, it is recommended to take a swab for COVID-19.

\section{Sleep Disorders Center Preparedness (Specific Considerations)}

Sleep disorders center preparedness encompasses logistics (healthcare resources) and staff education and training to provide optimal services without compromising infection control guidelines.

\subsection{Infection Control}

Each sleep disorders center should ensure that a designated staff member, usually a clinic coordinator, is responsible for regularly monitoring COVID-19 updates from the state, local health departments, and the hospital managerial body to coordinate demands and response [8, 17]. In-person visits should be minimized by utilizing telemedicine or other 
online portals for pre-procedure counseling and education, screening and obtaining informed consent.

Within the center, physical distancing is to be maintained by spacing the chairs (1-2 meters), minimizing the waiting time in the waiting area, and keeping a low patient volume $[8,14]$. Waiting in vehicles, in the parking lots/areas, rather than in the center, should be encouraged for patient attendants. All these instructions should be passed to the patients beforehand through telemedicine portals or phone calls [4, $8,15]$.

Additionally, patient attendants and visitors are to be minimized, best to be avoided. For translation services, if needed, consider using remote-access services $[8,15]$. It is recommended to place visual alert posters and placards about COVID-19 signs and symptoms, hand hygiene, respiratory hygiene, and cough etiquettes at entrances and in strategic places [8]. Supplies for hygiene, such as alcohol sanitizers and sinks to wash hands, should be accessible and available to the staff and visitors $[8,17]$.

Staff should be educated and trained to follow infection control protocols and procedures for cleaning and inspecting all patient related equipment. For cleaning reusable equipment, the manufacturer guidelines and infection control guidelines should be strictly followed [19-21]. Cleaning of reusable equipment with mild household detergent is recommended; for disinfection 70-90\% isopropyl alcohol, $8.25-10 \%$ chlorine bleach, or $2 \%$ glutaraldehyde besides less popular options like heat sterilization and hydrogen peroxide gas plasma can be used. However, following manufacturers' guidelines is always the first choice $[17,18$, 22-24]. The reusable equipment or its components should be removed from the services for $72 \mathrm{~h}$ before the next use, after following the disinfection procedure. For contact surfaces like bedposts and tables, after cleaning for any visible contamination with soap water/soap soaked cloth, disinfection with ethanol 70-90\%, chorine based products $(0.1-0.5 \%)$, or $0.5 \%$ hydrogen peroxide is recommended [22, 24-27]. However, institutional infection control guidelines are to be considered [22, 24-27].

\subsection{Personal Protective Equipment (PPE)}

It is essential that clinicians, staff, and patients wear surgical masks while in the facility. PPE supplies need to be always available in the facility. While handling any aerosol-generating procedure such as the application of positive airway pressure (PAP) titration, full PPE is recommended. If carried out in a COVID-19 suspect or confirmed case, under emergent conditions, it warrants a negative pressure room beside the rest of the preparation. A prior fit-test for 95 respirators is highly recommended [6, 8, 10, 28-32]. Moreover, staff should be trained in the donning and doffing procedure for PPE use.
It is essential to monitor and secure PPE supplies actively. In addition to practicing judicious use, it should be ensured that the staff is educated about the indications of use, i.e., when and where the use of PPE is indicated like handling aerosol-generating procedures or cleaning used sleep study equipment. The clinicians, staff, and patients should use surgical masks in the center [8, 17, 32].

\section{A Stepwise Approach to the Reopening of the Sleep Disorders Centers, Amid Covid-19 Pandemic}

The first principle is to postpone and reschedule all sleep studies for the patients who are COVID-19 positive or COVID-19 suspect. Moreover, there should be no penalties for patient cancellations and missed appointments related to respiratory illness, and rescheduling should be readily available to encourage patients to declare symptoms $[2,8,16]$.

For the high epidemic areas, sleep studies are to be suspended, except in case of emergency. For critically ill patients with sleep-disordered breathing, diagnostic and therapeutic studies should be commenced after a careful COVID-19 screening and thorough consideration of the potential benefits and risks $[2,8,11,15,16]$.

In this scenario of high epidemic areas, there is large scale community transmission, healthcare staffing is significantly impacted, and multiple cases within the community are reported daily. Therefore, it is recommended to postpone and reschedule polysomnography (PSG) for children and adults except in an emergency. Wherever testing for COVID19 infection is available, and all patients can be tested in a reasonable timeframe, all patients should have symptom screening through phone before arrival and upon arrival in the sleep center $[2,8,12,13]$.

Due to the high risk of aerosolization, titration studies and split-night studies should be postponed except in highly emergent conditions. In the latter setting, PAP administration should be taken in a negative pressure room (when feasible) and ensure that the technologist uses the appropriate PPE. Due to the high risk of aerosolization, PAP devices should not be operated in the clinical rooms even for demonstration [5, 6, 8, 12, 15, 28]. Figure 1 demonstrates a stepwise approach to the reopening of the sleep disorders center, taking into consideration the rate of infection in the community and the urgency of the sleep study.

Moreover, all in-person routine appointments should be postponed and rescheduled. The American Academy of Sleep Medicine (AASM) recommends, and many other societies endorse, that sleep clinics should consider deferring all elective, in-person sleep clinic appointments [8, $11,15,18]$. However, telemedicine contact is encouraged to avoid loss of follow up and patient confidence. For 


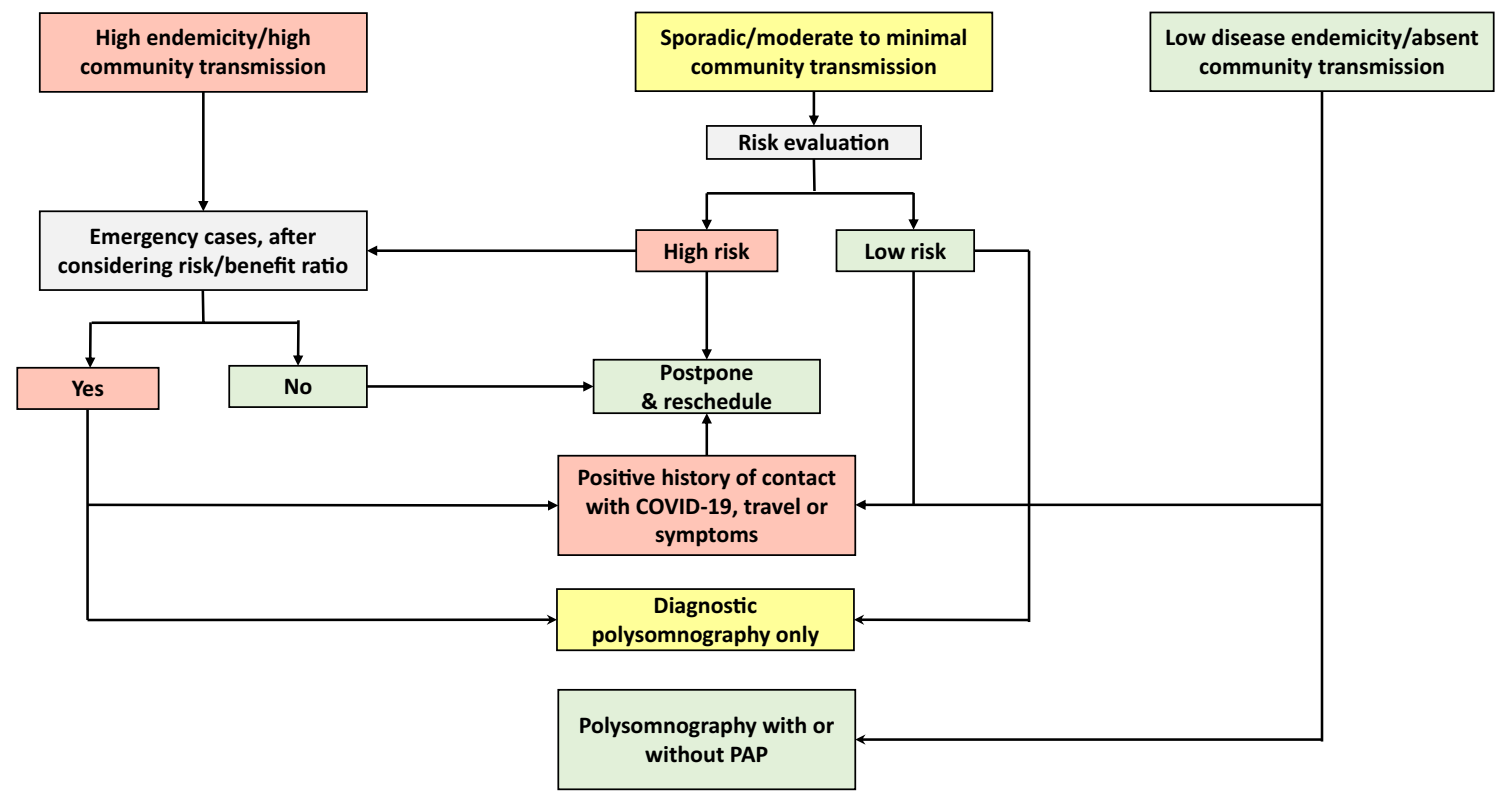

Fig. 1 A stepwise approach to the reopening of the sleep disorders center, taking into consideration the rate of infection in the community and the urgency of the sleep study

emergency or unavoidable in-person visits, infection control protocols and guidelines are to be followed and recommended PPE to be used $[8,15,18]$. The number of people present in the sleep disorders center at any time should be minimized; therefore, patient attendants and visitors should be prohibited. However, if indispensable, they need to be pre-screened like patients. If a language translator is required, remote-access translator services should be availed [8, 15, 32].

For the areas with only sporadic cases or small clusters, which corresponds to minimal to moderate community transmission, sleep studies can be continued after weighing the risk-benefit ratio [2, 8, 15]. However, the general agreement between different societies, in view of the epidemiological possibility of multiple cases of COVID-19 in the community is:

- Postpone and reschedule in-laboratory administration of PAP therapy except in emergencies; however, the same can be duly considered if the center can spare a negative pressure room for the procedure, in case of emergency.

- Always avoid PAP device use (even demonstration in the clinic setting). For home use of PAP therapy, the reader is referred to a recently published paper about PAP therapy during the COVID-19 pandemic [6].

- Postpone all PSG (diagnostic and therapeutic) in all the patients who are at a higher risk of getting severe COVID-19 illness, such as older adults, pregnant women, and patients with underlying cardiac, renal, or metabolic disorders or patients who are immunocompromised [33].
- Consider, carefully, to resume diagnostic in-lab sleep studies for the patients who are not at higher risk of getting severe COVID-19 illness, like adults (without comorbidities) and children [33].

- Resume in-person clinical consultations for the patients who are not at higher risk of severe COVID-19 illness, only if telemedicine consultations are unavailable or suboptimal, [33].

- Visitors/attendants should be limited, but if found indispensable, they need to be pre-screened like patients [6, $8,11,16]$.

However, it is to be emphasized here that sleep studies are to be suspended for subjects with the following conditions in the past 2 weeks: travel or residential history in the high epidemic area, contact history with COVID-19 infected patients, contact history with febrile patients or subjects with respiratory tract symptoms from the high epidemic or cluster outbreak area, reported fever or respiratory tract symptoms and recovered patient who does not have negative PCR test $[2,8]$.

In the areas where disease endemicity is low or community transmission is absent or minimal, resumption of routine in-laboratory diagnostic as well therapeutic studies for both adults and children can be done, in addition to PAP use in the clinic setting $[2,8,16]$. In addition, in-person appointments can be restarted as needed, including clinic visits and PAP setup demonstrations. Additionally, HSAT can be resumed following the usual protocols and instructions (restriction is no longer warranted as previously described). 
Table 2 A summary of the recommendations for home sleep apnea test

Home sleep apnea test recommendations

1. Use a fully disposable device or at least its disposable components.

2. The choice of a model that ensures home delivery.

3. Instructions for use and safe handling to be made available remotely or through brochures in the package.

4. The packages to be delivered with 'sterilization-status' stamped tags.

5. The reusable devices or its components are to be cleaned and sanitized before next use, preferably to be removed from the service for $72 \mathrm{~h}$ before the next use.

6. Cleaning and sterilization to be done by the staff with full PPE.

Nevertheless, continuous monitoring of state and local public health communication for warnings of any increase in community transmission is mandatory. Moreover, practical emphasis on patient screening and reviewing local epidemiological dynamics is highly recommended; any patient with confirmed or suspected COVID-19 infection is to be postponed. Under emergency conditions, remote control multimodal ventilator, wherever available, is suggested to ensure healthcare worker safety. For patients who have recovered from COVID-19 infection, there should be strong evidence of negative results of nucleic acid testing before taken for the sleep study, diagnostic as well as therapeutic; otherwise, it is best to be postponed $[2,5,8,11,15,16]$.

\section{Home Sleep Apnea Testing (HSAT) Service}

HSAT has been successfully used for years to diagnose patients with moderate to a high likelihood of OSA. OSA is a serious health problem that can have serious health consequences and even fatal outcomes such as cardiac complications, hypertension, and stroke. Therefore, during the COVID-19 era, HSAT has been proposed as a solution to avoid the delay in diagnosing and treating patients with OSA $[3,34,35]$. Table 2 shows a summary of the guidelines for HSAT.

It is recommended to consider single-use, fully disposable devices, and its components when feasible. For HSAT, level 3 cardiopulmonary monitoring devices are usually used $[8$, $11,15,16]$.

The choice of the HSAT model should take into consideration the need to ensure a home-delivery so that patients do not need to attend the center in-person to receive or return the device, nor a technician needs to visit the patient to complete the hook-up process. Therefore, patients should be provided with instructions/guidance to ensure proper setup and safe handling of the package prior to the delivery date through brochures, online videos, or telemedicine consultations $[8,13,16]$. We recommend that HSAT devices should be delivered in sealed packages with a 'sterilization-status' stamped tags to avoid medicolegal issues. When reusable devices are used, the units must be cleaned and sanitized according to infection control guidelines and manufacturer's instructions. It would be best to remove the reusable devices from service for at least $72 \mathrm{~h}$ after use in addition to disinfection before its next use [8, 17]. It is recommended as well that individuals responsible for handling and cleaning reusable HSAT devices after use must wear appropriate PPE $[8,32]$.

\section{Summary}

COVID-19 mandates a radical change in healthcare policies and procedures to accommodate exigencies and contingencies of the pandemic. To resume the normal or near-normal functioning of sleep disorders centers, we need to weigh and consider day-to-day epidemiological situational reports to tailor the services accordingly. It is mandatory to follow infection control guidelines without compromise. Resumption plans and strategies warrant interdisciplinary deliberations and appraisals.

Funding This work was supported by the Strategic Technologies Program of the National Plan for Sciences and Technology and Innovation in the Kingdom of Saudi Arabia (MED511-02-08). The study sponsors played no role in the study design, the collection, analysis or interpretation of data, writing the manuscript, or the decision to submit the manuscript.

\section{Compliance with ethical standards}

Conflict of interest The authors have no conflict of interest to declare.

\section{References}

1. Bi Q, Wu Y, Mei S, Ye C, Zou X, Zhang Z, Liu X, Wei L, Truelove SA, Zhang T, Gao W, Cheng C, Tang X, Wu X, Wu Y, Sun B, Huang S, Sun Y, Zhang J, Ma T, Lessler J, Feng T. Epidemiology and transmission of COVID-19 in 391 cases and 1286 of their close contacts in Shenzhen, China: a retrospective cohort 
study. Lancet Infect dis. 2020. https://doi.org/10.1016/s1473 -3099(20)30287-5.

2. Zhang XL, Xiao Y. Sleep health service in China during the COVID-19 outbreak. J Clin Sleep Med. 2020. https://doi. org/10.5664/jcsm.8472.

3. World Health Organization. Coronavirus disease (COVID-19) pandemic. 2020. https://www.who.int/emergencies/diseases/novel -coronavirus-2019. Accessed on 16 June 2020.

4. Grote L, McNicholas WT, Hedner J. Sleep apnoea management in Europe during the COVID-19 pandemic: data from the European Sleep Apnoea Database (ESADA). Eur Resp J. 2020;55(16):2001323. https://doi.org/10.1183/13993003.01323 -2020 .

5. Krishnan V. COVID-19 and home positive airway pressure (PAP) therapy. Am J Resp Crit Care Med. 2020;201(12):P28-P29. https ://doi.org/10.1164/rccm.2020c6.

6. Pirzada AR, Aleissi SA, Almeneessier AS, BaHammam AS. Management of aerosol during noninvasive ventilation for patients with sleep-disordered breathing: important messages during the COVID-19 pandemic. Sleep Vigil. 2020. https://doi.org/10.1007/ s41782-020-00092-7.

7. Pazarl AC, Ekiz T, Lik F. Coronavirus disease 2019 and obstructive sleep apnea syndrome. Sleep Breath. 2020. https://doi. org/10.1007/s11325-020-02087-0.

8. The American Academy of Sleep Medicine. COVID-19 mitigation strategies for sleep clinics and centers-REOPENING. The American Academy of Sleep Medicine. 2020. https://aasm.org/ covid-19-resources/covid-19-mitigation-strategies-sleep-clinicslabs. Accesed on 12 June 2020. Accessed 15 June, 2020.

9. Centers for Disease Control and Prevention. Overview of testing for SARS-CoV-2. 2020. https://www.cdc.gov/coronavirus/2019ncov/hcp/testing-overview.html. Accessed on 14 June 2020.

10. Centers for Disease Control and Prevention. Interim infection prevention and control recommendations for patients with suspected or confirmed coronavirus disease 2019 (COVID-19) in healthcare settings. Centers for Disease Control and Prevention. 2020. https:// www.cdc.gov/coronavirus/2019-ncov/hcp/infection-control-recom mendations.html. Accessed on 16 June 2020.

11. Ayas NT, Fraser KL, Giannouli E, Hanly PJ, Kendzerska T, Katz SL, Lachmann BN, Lajoie A, Minville C, Morrison D, Narang I, Povitz M, Skomro R, Spurr KF. Helping Canadian health care providers to optimize Sleep Disordered Breathing management for their patients during the COVID-19 pandemic. Canadian J Resp Crit Care Sleep Med. 2020. https://doi.org/10.1080/24745 332.2020.1758442.

12. Rothe C, Schunk M, Sothmann P, Bretzel G, Froeschl G, Wallrauch C, Zimmer T, Thiel V, Janke C, Guggemos W, Seilmaier M, Drosten C, Vollmar P, Zwirglmaier K, Zange S, Wölfel R, Hoelscher M. Transmission of 2019-nCoV infection from an asymptomatic contact in Germany. N England J Med. 2020;382(10):9701. https://doi.org/10.1056/nejmc2001468.

13. The American Academy of Sleep Medicine. COVID-19 and Testing for Sleep Apnea. American Academy of Sleep Medicine. 2020. https://www.sleepassociation.org/covid-19-and-sleep-apnea -testing/ Accessed on 17 June 2020.

14. World Health Organization. Coronavirus disease (COVID-19) advice for the public. World Health Organization. 2020. https:// www.who.int/emergencies/diseases/novel-coronavirus-2019/advic e-for-public. Access on 13 June 2020.

15. British Thoracic Society. COVID-19: information for the respiratory community. British Thoracic Society. 2020. www.britthoracic.org.uk/about-us/covid-19-information-for-the-respirator y-community. Accessed on 15 June 2020.

16. Australian Sleep Association. Updated recommendations for polysomnography during CoVID-19 pandemic: recommencement of positive airway pressure (PAP) therapy studies. 2020. https://
www.sleep.org.au/common/Uploaded\%20files/Public\%20Files/ COVID-19\%20docs/FINAL\%20PAP\%20psg\%20recommencemen t_280520.pdf. Accessed on 280513 June 282020.

17. Centers for Disease Control and Prevention. Infection Control Guidance for Healthcare Professionals about Coronavirus (COVID-19). Centers for Disease Control and Prevention. 2020. https://www.cdc.gov/coronavirus/2019-ncov/hcp/infection-contr ol.html. Accessed on 11 June 2020.

18. European Centre for Disease Prevention and Control. Infection prevention and control and preparedness for COVID-19 in healthcare settings-third update. ECDC. 2020. https://www.ecdc.europ a.eu/en/publications-data/infection-prevention-and-control-andpreparedness-covid-19-healthcare-settings\#copy-to-clipboard. Accessed on 17 June 2020.

19. Philips. Clinical support \& resources for Philips ventilators and respiratory products. Philips. 2020. https://www.philips.sa/en/ healthcare/medical-specialties/covid-19/sleep-and-respirator y-care-covid-19/clinical-resource-and-training-page\#triggernam e=menu_resources. Accessed on 20 June 2020.

20. GRASS Technologies. Genuine Grass Gold Disc Electrode Care. 2011. https://bio-medical.com/media/support/genuine_grass _gold_disc_electrode_care.pdf. Accessed on 20 June 2020.

21. NONIN. Model 8000SX, 8000SX-WO, 8000SX-WO2 Reusable Soft Pulse Oximeter Sensors. 2016. https://www.nonin.com/wpcontent/uploads/Operators-Manual-8000S.pdf. Accessed on 8020 June 2020.

22. Center for Disease Control and Prevention. Disinfection and Sterilization. CDC. 2020. https://www.cdc.gov/infectioncontrol/guide lines/disinfection/tables/table1.html. Accessed on 18 June 2020.

23. Philips for cystic fibrosis community care COVID-19 cleaning $\&$ disinfection guidance for homecare providers. https://cfcc.org. au/wp-content/uploads/2020/2003/COVID-2019-Philips-Guida nce_D2002.pdf. Accessed on 2018 June 2020.

24. World Health Organization. Cleaning and disinfection of environmental surfaces in the context of COVID-19. WHO. 2020. www. who.int/publications/i/item/cleaning-and-disinfection-of-envir onmental-surfaces-inthe-context-of-covid-19. Accessed on 19 June 2020.

25. World Health Organization. Coronavirus disease (COVID-19) technical guidance: Infection prevention and control/WASH. WHO. 2020. https://www.who.int/emergencies/diseases/novel -coronavirus-2019/technical-guidance/infection-prevention-andcontrol. Accessed on 18 June 2020.

26. Centers for Disease Control and Prevention. Cleaning and Disinfecting Your Facility. CDC. 2020. https://www.cdc.gov/coron avirus/2019-ncov/community/disinfecting-building-facility.html. Accessed on 17 June 2020.

27. Ong SWX, Tan YK, Chia PY, Lee TH, Ng OT, Wong MSY, Marimuthu K. Air, surface environmental, and personal protective equipment contamination by severe acute respiratory syndrome coronavirus 2 (SARS-CoV-2) from a symptomatic patient. JAMA. 2020;323(16):1610-2. https://doi.org/10.1001/ jama.2020.3227\%JJAMA.

28. Ferioli M, Cisternino C, Leo V, Pisani L, Palange P, Nava S. Protecting healthcare workers from SARS-CoV-2 infection: practical indications. Eur Respir Rev. 2020;29(155):200068. https://doi. org/10.1183/16000617.0068-2020.

29. Bartoszko JJ, Farooqi MAM, Alhazzani W, Loeb M. Medical masks vs N95 respirators for preventing COVID-19 in healthcare workers: a systematic review and meta-analysis of randomized trials. Influenza Other Respir Viruses. 2020;14:365-73. https:// doi.org/10.1111/irv.12745.

30. Verbeek JH, Rajamaki B, Ijaz S, Sauni R, Toomey E, Blackwood B, Tikka C, Ruotsalainen JH, Kilinc Balci FS. Personal protective equipment for preventing highly infectious diseases due to exposure to contaminated body fluids in healthcare staff. 
Cochrane Database Systematic Rev. 2020;4(4):CD011621. https ://doi.org/10.1002/14651858.cd011621.pub4.

31. Smith JD, MacDougall CC, Johnstone J, Copes RA, Schwartz B, Garber GE. Effectiveness of N95 respirators versus surgical masks in protecting health care workers from acute respiratory infection: a systematic review and meta-analysis. CMAJ. 2016;188(8):56774. https://doi.org/10.1503/cmaj.150835.

32. World Health Organization. Rational use of personal protective equipment (PPE) for coronavirus disease (COVID-19): interim guidance, 19 March 2020. (WHO/2019-nCoV/IPC PPE use/2020.2). 2020. https://apps.who.int/iris/handle/10665/33149 8. License: CC BY-NC-SA 331493.331490 IGO. Accessed on 331418 June 332020.

33. Clark A, Jit M, Warren-Gash C, Guthrie B, Wang HHX, Mercer SW, Sanderson C, McKee M, Troeger C, Ong KL, Checchi F, Perel P, Joseph S, Gibbs HP, Banerjee A, Eggo RM, Nightingale ES, O'Reilly K, Jombart T, Edmunds WJ, Rosello A, Sun FY, Atkins KE, Bosse NI, Clifford S, Russell TW, Deol AK, Liu Y, Procter SR, Leclerc QJ, Medley G, Knight G, Munday JD, Kucharski AJ, Pearson CAB, Klepac P, Prem K, Houben
RMGJ, Endo A, Flasche S, Davies NG, Diamond C, van Zandvoort K, Funk S, Auzenbergs M, Rees EM, Tully DC, Emery JC, Quilty BJ, Abbott S, Villabona-Arenas CJ, Hué S, Hellewell J, Gimma A, Jarvis CI. Global, regional, and national estimates of the population at increased risk of severe COVID-19 due to underlying health conditions in 2020: a modelling study. Lancet Global Health. 2020;2214-2109X(2220):30263-4. https://doi. org/10.1016/s2214-109x(20)30264-3.

34. ResMed. COVID-19 resource center. ResMed. 2020. https:// www.resmed.com/us/en/healthcare-professional/covid-19.html. Accessed on 18 June 2020.

35. ResMed. COVID-19: our response. Resmed. 2020. https://www. resmed.com/au/en/healthcare-professional/covid-19-our-response. html. Accessed on 16 June 2020.

Publisher's Note Springer Nature remains neutral with regard to jurisdictional claims in published maps and institutional affiliations. 